\title{
LA COMPRENSIÓN DE LAS ORGANIZACIONES PRODUCTORAS DE SERVICIOS SOCIALES DESDE EL PARADIGMA DE LOS SISTEMAS COMPLEJOS
}

\author{
María Lorena Molina M. * \\ Nidia Esther Morera G. *
}

\section{Introducción}

I as organizaciones que tienen como razón de existencia producir servicios sociales han sido diseñadas como organizaciones burocráticas, caracterizadas por la especialización, la división funcional de trabajo, la jerarquía de autoridad y de la comunicación, la aplicación de reglas, la separación entre lo técnico y lo político, y con enfoques de administración en los que prevalecen criterios clásicos y neoclásicos. No obstante, cuando tales organizaciones se constituyen en objetos de estudio, el aspecto desafiante de la investigación resulta ser las interacciones del sistema organizacional, lo cual implica penetrar básicamente tres dimensiones interactuantes entre sí y en su interior: macrocontexto, ambiente de tarea y microambiente. En tales dimensiones los actores del sistema, externos e internos, constituyen relaciones, concepciones y acciones contradictorias, que conducen a interrogarse sobre cuál es la verdadera misión organizacional, más allá de la expresada en el texto oficial y cuáles son los estratos y diversos intereses de quienes toman decisiones político-estratégicas y además de quienes

* Profesoras Investigadoras de Postgrado Gerencia Social, Universidad de Costa Rica. 
constituyen la categoría Población - Meta. También es necesario interrogarse sobre cuáles son las fuerzas generadoras de los antagonismos a los consensos, que se derivan de las características de las personas miembras, del tipo de diseño organizacional y la relación entre los métodos y demandas, que hace posible un proceso de trabajo que transforma insumos en resultados.

Este trabajo se ocupa de ofrecer a los lectores una síntesis teórica de la perspectiva de la complejidad de los sistemas, para comprenderla en sus orígenes, premisas actuales y su pertenencia en términos de un campo disciplinario en construcción llamado Gerencia Social; y al mismo tiempo ofrecer, una argumentación acerca de por qué las organizaciones productoras de servicios sociales son sistemas complejos.

\section{Sobre el origen y la constitución de la teoría de sistemas}

En los años 20, se identifica el germen de la teoría general de sistemas como un método para el estudio de sistemas biológicos, que permitiera superar el abordaje de las partes y los procesos en forma atomizada y encararlos identificando la coordinación entre ellos. En su origen este método de investigación se llamó "biología organísmica” y la teoría explicativa se denominó Teoría de Sistemas del Organismo; luego se sustituyó el término ORGANISMO por el de ENTIDADES ORGANIZADAS y ello incluyó la personalidad, los grupos, las organizaciones, entre otros.

En los años 30, los avances teóricos en este campo identifican descripciones de propiedades de los sistemas entre las que destacan: la totalidad, la diferenciación, la finalidad, la equifinalidad, el crecimiento y la competición. Bertalanffy ${ }^{1}$ en la década del cincuenta, esboza la Teoría de Sistemas Dinámicos y subraya que la idea de sistema no es una moda efímera o una reciente técnica, sino que este concepto es situable en la historia de las ideas (Bertalanffy y otros, 1987: 29-53).

1 Bertalanffy, nacido en 1901, austríaco reconoce la influencia del neopositivismo en su proceso de formación, sin que haya sido determinante. Otras influencias proceden del grupo berlinés de la sociedad filosófica empírica. Su formación corresponde a la época de la coexistencia de distintas corrientes filosóficas y políticas. Además de las citadas, el materialismo histórico y el nacional socialismo. 
En este sentido, es importante recordar que la noción antigua de sistema encuentra sus raíces en el pensamiento aristotélico, concretamente en la expresión "el todo es más que la suma de las partes" y en los conceptos de orden e intencionalidad. Bertalanffy en el texto citado se refiere a que el concepto de orden jerárquico de los sistemas se atribuye al pensamiento místico cristiano y la noción de lucha de las partes en una totalidad se identifica con Nicolás de Cusa; también señala que, en el pensamiento de Hegel se encuentra el concepto de la estructura dialéctica y de coincidencia de los contrarios y además otros pensadores como Köhler de la corriente Gestald, plantea la relación entre el todo y las partes; y que Kotka se refiere a las comunidades como sistemas.

Esta comprensión totalizadora de los sistemas de origen aristotélica -siguiendo a Bertalanffy y otros (1987)- es opacada en el siglo XVI y XVII con la revolución científica, cuyo paradigma enfatizó la fragmentación de todo problema en tantos elementos simples y separados como fuera posible -según lo planteó Renato Descartes y Bacon-, la descripción y el análisis de los hechos con métodos matemáticos, apoyados en la experimentación y la observación. Así, como lo indica el mismo Bertalanffy, el racionalismo y el empirismo dominaron durante estos dos siglos e influyeron en forma relevante en los siglos siguientes con el desarrollo del Positivismo en las ciencias humanas, fundamentalmente con los aportes del Círculo de Viena, al concluir los años veinte.

El funcionalismo como corriente de pensamiento, identifica la noción de totalidad con una base organicista, lo cual significaba que el fenómeno interesa según la posición que ocupa y las funciones que realiza. En otras palabras, interesa la parte funcionalmente relacionada con las demás partes, no el análisis del conjunto, su génesis y el desarrollo de la totalidad. Las relaciones entre las partes manifiestan una interdependencia equilibrada y con igual peso, el sistema mantiene sus límites frente al medio; la tensión es un factor perturbador ocasional o contingente y el equilibrio se refiere a describir estados constantes. El análisis global es ahistórico y no interesa el análisis del conjunto (Díaz Polanco, 1979).

El método de las ciencias naturales se aplicó en las ciencias sociales como requisito de cientificidad; sin embargo los resultados de aplicar tal método no resolvieron problemas en los que intervienen múltiples relaciones, en tanto que lo que interesa con este método 
positivista es fragmentar, atomizar las partes y no captar sus interacciones. A raíz de esta limitante surgieron interrogantes referidas a la organización de los sistemas vivos y sobre la intencionalidad - discrecionalidad de éstos. Ditthey en el siglo XIX estableció las diferencias entre las ciencias naturales y las ciencias del espíritu o humanas y subrayó que éstas deben comprender el mundo, o sea, interpretar los procesos y que para ello, la hermenéutica es el método, lo cual sentó las bases del pensamiento weberiano y la sociología comprensiva (Bertalanffy y otros, 1987).

Marx recupera de Hegel la noción de la dialéctica y la integra con la concepción histórico materialista y el concepto de praxis. Desde el pensamiento marxista -afirma Díaz Polanco (1979)- interesa la comprensión del conjunto y sus transformaciones, como paso previo al entendimiento de las partes; el origen y el desarrollo del sistema, sus interdependencias contradictorias de pesos desiguales, son asuntos de relevancia esencial. El sistema social está en autodesarrollo y en interacción contradictoria con sus componentes internos y entre estos y los componentes externos. Interesan los aspectos macro del sistema social capitalista.

Diferentes disciplinas han enfocado con una visión mecanicista los fenómenos, centrando el interés en la parte y el proceso aislado. No obstante, esta visión tiende a superarse por una que no sólo estudia partes y procesos aislados, sino también resuelve problemas hallados en la organización y en la interacción dinámica de las partes. La Teoría General de los Sistemas se ocupa de la formulación y derivación de aquellos principios que son válidos para los sistemas en general y se opone al concepto MECANICISTA, LINEAL, CAUSAL una visión ORGÁNICA del mundo. Existen modelos, principios y leyes aplicables a sistemas generalizados o a sus subclases, sin importar el género, la naturaleza de sus elementos componentes y las relaciones o fuerzas que imperen entre ellos. Para esta teoría el problema consiste en saber qué puede decirse de sistemas materiales, informacionales y conceptuales, por ello se afirma que es polifónica en tanto tiene aplicación en diversos campos como la matemática, la ingeniería, la computación y las ciencia sociales. Su punto focal es el estudio de las interacciones como respuesta a una comprensión de los fenómenos complejos.

Klir $(1987: 17,18)$ plantea que existen tres enfoques (Mesarovic, Wymore y Klir) en el estudio de los sistemas; cuyos rasgos fundamentales son: 
a) La teoría axiomática o de Mesarovic, la cual es deductiva y se planteó a principios de los años sesenta. Explica la conducta de los sistemas según la especificación terminal o causal (entradas - salidas), el énfasis está en especificar el proceso de la conducta orientada por objetivos.

b) El enfoque de Wymore dirige la atención -según Klir- a definir el sistema basado en las estructuras de transición de los estados del sistema y los acoplamientos de los sistemas.

c) Un tercer enfoque de la Teoría de Sistemas es el inductivo, el cual según Klir, su foco de atención está en identificar las características de los sistemas y los problemas asociados a ellos. Aporta un espectro de cinco definiciones de sistemas y con ello sienta las bases para una metodología. De acuerdo con Klir un sistema cambia si cualquiera de las características primarias que entran en su definición cambia.

Robert A. Orchard (1987: 237-288) hace aportes para estudiar el proceso evolutivo de los sistemas al señalar los conceptos de autoorganización y autorreproducción. También señala que en el área de Teoría de Sistemas existen: teorías orientadas hacia sistemas generales (teorías matemáticas de sistemas abstractos); teorías de sistemas que generalizan rasgos y son aplicables a muchos sistemas de varias disciplinas y, teorías de sistemas específicos o especiales orientadas hacia una disciplina, con un nivel más bajo de generalización y un gran contenido específico. Esta última teoría señala el siguiente procedimiento metodológico para el estudio de sistemas desde una disciplina.

En general se debe tener claro que en el área de la Teoría de Sistemas se identifican los aportes de Mesarovic y Wymore para una teoría formal según Klir (1987); los aportes de orden metodológico elaborados por Ashly, Klir y Orchard, como destaca Bertalanffy (1987), y los aportes de orden epistemológico los encontramos en Buckley (1987), Bertalanffy (1987) y Morin (1995), quienes desarrollan con sus teorías una nueva forma de pensar y mirar el mundo. 


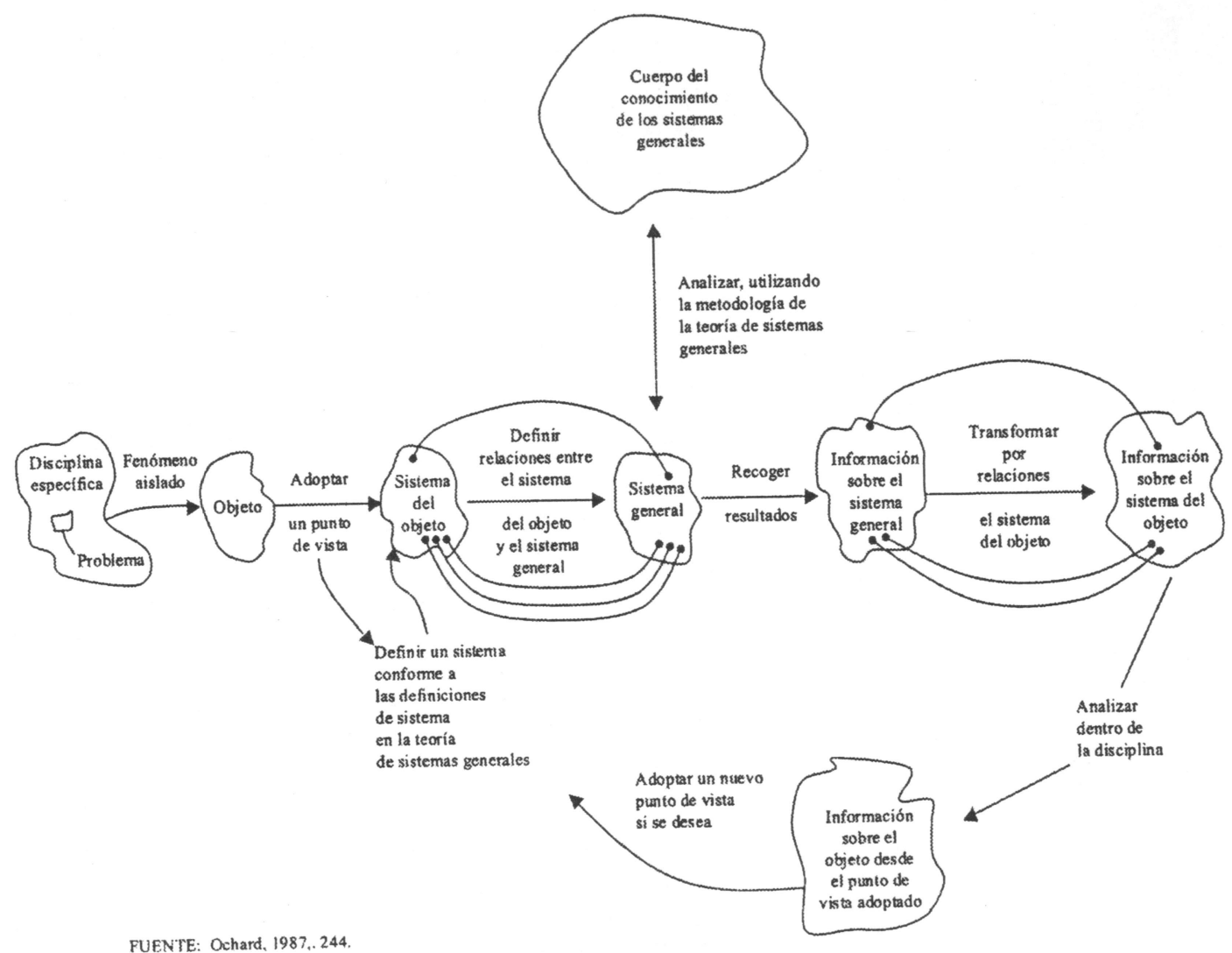




\section{La epistemología de la complejidad}

Los principales aportes innovadores de la Teoría de Sistemas se refieren a observar el mundo como conjunto de fenómenos interrelacionados, de aquí que la complejidad se constituya en una categoría relevante para la comprensión de un sistema. Por otra parte, al estar interrelacionados los fenómenos, ciertos conceptos y métodos no dependen exclusivamente de la naturaleza específica del campo disciplinario, ello hace que surjan lazos entre distintas disciplinas.

La causalidad lineal (descomponer el objeto en unidades elementales) es insuficiente para captar la característica esencial de los sistemas vivos. Esto desafía la búsqueda de otra epistemología pues la "ciencia normal" está construida para manejar relaciones insertas en sistemas. Bertalanffly ha subrayado que el carácter fundamental de un objeto viviente es su organización de manera que un examen de las partes y de los procesos aislados no puede dar una explicación totalizadora de los fenómenos vitales, pues no informa acerca de la coordinación de las partes y sobre los procesos.

Las entidades organizadas (los grupos, las instituciones, la personalidad, los ingenios tecnológicos) llevan a recuperar la máxima aristotélica el TODO es más que la suma de las partes en tanto que la comprensión de un todo organizado requiere conocer tanto sus partes, como sus interrelaciones. Entre las tendencias de la teoría general de sistemas como nuevo paradigma, la filosofía de los sistemas subraya la reorientación del pensamiento y de la concepción del mundo. La percepción es una resultante de la interacción entre lo conocido y el que conoce y por tanto dependiente de una multiplicidad de factores biológicos, psicológicos, culturales y lingüísticos. En las últimas décadas el trabajo de neurofisiólogos, psicólogos y lingüistas plantea que la organización innata del sistema nervioso interviene en la estructuración de la percepción del pensamiento conceptual, esto nos aproxima a un proceso constructivo más que de reproducción. Al respecto Buckley W. (1987: 225) plantea que "el observador no es simplemente receptor y registrador de las señales incidentes y de datos sensoriales, sino que activamente aporta información adicional, así como ayuda a construir el armazón u organización del conjunto de referencia correspondiente al conocimiento interno, conjunto que da sentido a las señales adicionales generadas desde el exterior o desde el interior, por ejemplo, el pensamiento o la emoción. 
La ontología de los sistemas, o sea lo que se entiende por sistema y como éste se da en los distintos niveles del universo de observación, lleva a distinguir entre sistemas reales (entidades percibidas mediante la observación o inferidas de éste y con una existencia independiente del observador) y sistemas conceptuales que son en esencia construcciones simbólicas (música, matemáticas). "Un sistema es definible por su cohesión en un sentido amplio, esto es, por las interrelaciones entre los elementos componentes. Las interrelaciones no se perciben directamente, son construcciones conceptuales" (Bertalanffy, 1987: 48).

Otro aspecto del que se ocupa la filosofía de sistemas se refiere a las relaciones entre el ser humano y el mundo, con lo cual Bertalanffly reafirma las preocupaciones humanistas de su Teoría General de Sistemas. Ello marca una clara diferencia con los teóricos de sistemas, con orientaciones "mecanicistas" que dan origen a la crítica centrada en la falsa sinonimia "Teoría de Sistemas es sociedad tecnocrática y devaluación del hombre”.

Otras tendencias de la teoría general de sistemas como nuevo paradigma son:

a) La ingeniería de sistemas que da respuestas a problemas de la tecnología y de la sociedad p.e: ecosistemas; organizaciones formales; sistemas socioeconómicos, y

b) La ciencia de los sistemas que comprende el desarrollo de teorías de los sistemas en las distintas ciencias. Sus métodos se refieren a la descripción interna referida a la estructura y las interdependencias (teoría clásica de sistemas) y la descripción externa (teoría dinámica de los sistemas) referida a la interacción con el medio.

Bertalanffy señala que definir el sistema supone identificar la direccionalidad y las relaciones. Los problemas de las ciencias biológicas, sociales y de la conducta humana merecen otro manejo, o sea referirlos a categorías de INTERACCION que superen la causalidad lineal. La Epistemología de los sistemas en esencia se ocupa de: qué se va a entender por sistema o sea su objeto; la interacción Observador - Objeto observado (real o conceptual); y de las relaciones entre el ser humano y el mundo o sea los valores. Para Leñero (1975) la Teoría General de Sistemas es un enfoque para el estudio científico de la realidad. Antes de la Teoría General 
de Sistemas, cada ciencia se preocupaba de aislar los fenómenos que le interesaba estudiar para desentrañar sus características esenciales y las relaciones de causa y efecto que las rigen. Sin embargo, la complejidad de la vida social hace insuficiente el conocimiento especializado por sí solo. De aquí la utilidad de esta teoría al enfocar el comportamiento de los elementos de la realidad frente a otros elementos ubicados en un contexto con el cual tiene múltiples interacciones de distinto nivel de relevancia. El grado de relevancia de las interrelaciones está dado por la contribución que ellas aportan a los elementos que en su conjunto producen un resultado característico y que justifican la participación de cada elemento en el conjunto.

El conjunto de elementos vinculados por las interrelaciones que producen un resultado es lo que se denomina SISTEMA. No todas las relaciones que pueden existir son significativas para un sistema sino sólo aquellas que conducen de alguna forma al logro del objetivo.

La Teoría General de Sistemas advierte de las relaciones vitales con el ambiente y que para el estudio de un sistema deben definirse sus componentes vitales: el objetivo, los elementos, las interrelaciones y el ambiente. Así es posible analizar:

a) CÓMO se relacionan determinados elementos y PARA QUÉ. la investigación parte de los elementos para descubrir el objetivo, el sistema y el ambiente..

b) CUÁles elementos son necesarios y CÓMO se interrelacionan para producir el OBJETIVO. Investigar el objetivo que se conoce permite descubrir el sistema que lo produce.

c) PARA QUÉ existen determinadas interrelaciones entre ciertos elementos y CUÁLES son los elementos involucrados. Investigar ciertos elementos interrelacionados ayuda a descubrir el sistema y su objetivo.

\section{Sobre los tipos de sistemas}

Bertalanffy (1987: 47) señala que los aportes de la Teoría General de Sistemas dan cuenta de la existencia de SISTEMAS REALES o entidades percibidas mediante la observación o inferidas de ésta, y con una existencia independiente del observador. También dan cuenta de la existencia de SISTEMAS CONCEPTUALES, 
que en esencia son construcciones simbólicas que corresponden a realidades.

Entre los sistemas reales y conceptuales se pueden identificar los abiertos y los cerrados.

Los sistemas cerrados son aquellos diseñados para reaccionar en forma perfectamente previsible ante los estímulos. No tiene posibilidad de cambiar por sí mismo sus reacciones en el medio si produjeran variaciones de naturaleza distinta para lo cual están diseñados; ejemplos:

a) Sistemas de estructuras rígidas (puentes, edificios).

b) Sistemas de mecanismos simples (reloj, máquinas simples).

c) Sistemas cibernéticos (termostato reguladores de velocidad).

Los sistemas abiertos son aquellos en los que hay vida, existe capacidad para reaccionar ante las variaciones del medio. La ciencia que estudia las relaciones de los sistemas abiertos con el ambiente es la cibernética. Ashby (citado en Rey, 1975) la definió como la ciencia que se ocupa de estudiar los sistemas de cualquier naturaleza capaces de recibir, conservar y transformar información y utilizarla para su propia dirección y regulación.

En el ser humano y en los sistemas sociales existe una capacidad adicional, si el ambiente no les es favorable, los sistemas superiores tienen la posibilidad de actuar sobre el ambiente para modificarlo conforme a sus necesidades e intereses. Bukley (citado en Rey, 1975) clasifica los sistemas en tres grupos:

a) Modelo de equilibrio corresponde a sistemas cerrados indiferentes del medio.

b) Modelo homeostático organísmico sistemas interactuantes con el medio pero con capacidad para adaptar el sistema al medio (Homeóstasis es un concepto de la biología que se aplica a la facultad que poseen los organismos vivos de mantener sus condiciones de equilibrio interno a pesar de las variaciones del ambiente).

c) Modelo procesal o de sistema adaptativo corresponde a los sistemas que al interactuar con el medio pueden adaptarse en él o bien son capaces de modificar el ambiente de acuerdo con sus necesidades o intereses. 
En los sistemas a lo largo del tiempo se observa la degradación paulatina de la capacidad para lograr su objetivo. En los sistemas abiertos este proceso es irreversible y se le llama ENTROPÍA. En los organismos vivos principalmente en al etapa de su desarrollo y madurez existe la capacidad interna de generar su recuperación y mantener la vida a pesar de su desgaste interno y a pesar de que se produzcan modificaciones en el ambiente. Al importar energía los sistemas generan la NEGAENTROPÍA que necesitan para contrarrestar los efectos de la entropía.

Rey (1975) señala que el concepto de SISTEMA aporta a la dirección de organizaciones un modelo para la interpretación del fenómeno organización en su dinámica y estructura al considerarla como un sistema abierto y vivo. Los modelos clásicos de Organización se concentran en los elementos y su funcionamiento en lo interno como si fueran independientes de los cambios en el entorno. Ejemplos: proceso de trabajo; estructura; relaciones humanas, proceso administrativo, entre otros. Dichos modelos tampoco permiten reconocer la equifinalidad de los sistemas o sea diversos caminos para obtener un mismo objetivo o bien la multifinalidad un mismo camino para la obtención de diversos resultados.

Los enfoques clásicos al no considerar las interacciones de la organización con su entorno, no permiten desarrollar la función de inteligencia que detecte los cambios en el entorno y dicha función depende de la calidad del subsistema de información que la organización posea, de éste depende el desarrollo y la supervivencia. De tal manera que la aplicación de la Teoría de Sistemas al campo de la comunicación y de la información también constituyen aportes relevantes para la comprensión ecosistémica de las organizaciones y para el desarrollo de la función gerencial.

\section{La teoría general de sistemas como sustento epistemológico de la gerencia de organizaciones productoras de servicios sociales}

La Teoría General de Sistemas sirve de sustento epistemológico a la Gerencia Social de Organizaciones Productoras de Servicios Sociales (OPSS), en tanto que éstas constituyen todos organizados complejos. Comprender tales organizaciones requiere de un paradigma en el cual es fundamental la noción de relación entre los elementos constitutivos de las tres dimensiones que configuran el sistema, a saber: la macrocontextual, la intermedia y la microorganizacional. 
La OPSS son sistemas complejos en tanto existen y se desarrollan a partir de un tejido de relaciones múltiples, diversas y marcadas por intencionalidades consensuadas a veces, antagónicas otras, o también complementarias. El entramado de las interrelaciones, para efectos explicativos, está configurado por niveles de relaciones según son las dimensiones del sistema.

Un primer nivel de complejidad puede ser identificado a partir de la reconstrucción de la OPSS y sus relaciones con actores tomadores de decisiones legales, políticas, presupuestarias. Tales interacciones delimitan el ámbito de competencias o misión y objetivos organizacionales con sus consecuentes valores. Tales vínculos adquieren una dinámica y direccionalidades acordes al proyecto político-ideológico que se configura en la relación Estado, Gobierno -sociedad civil. En este nivel de complejidad las OPSS construyen su legalidad, legitimidad y las fuentes de provisión de los recursos económicos y de información en términos de directrices políticas y a su vez, según sean sus bases de poder, influyen en la configuración de las relaciones de otras OPSS.

En la dimensión intermedia encontramos otro segundo nivel de complejidad o sea un conjunto organizacional interactuante de manera más frecuente con la organización productora de servicios sociales. En este conjunto, se identifican subconjuntos de proveedores de recursos, de legitimidad; subconjuntos de organizaciones competidoras en la consecución de los recursos o bien porque comparten el dominio organizacional (geográfico o por tipo de servicios) y encontramos también a la población - meta que manifiesta sus demandas en forma individual o colectivamente.

En la dimensión microorganizacional encontramos el tercer nivel de complejidad, éste es la expresión de intereses afines o antagónicos con los actores de la dimensión macroestructural e intermedia y la configuración de interrelaciones diversas entre los componentes o subsistemas sociotécnicos. Este tipo de organización que aquí nos ocupa, puede ser conceptualizado como un sistema abierto, dinámico y vivo, que intercambia energía con el medio ambiente. Puede ser estudiado con base en un conjunto de principios aplicables a todo tipo de sistema, tratando de identificar sus elementos y las interrelaciones entre ellos, y a su vez desde la especificidad disciplinaria de la Gerencia Social.

La utilidad de esta teoría estriba en que al definir que un sistema es un modelo de naturaleza general, esto es, una representación 
conceptual de ciertos caracteres universales de entidades observadas (Bertalanffy, 1987) y teniendo presente que el uso de modelos y construcciones representativas constituye el método general de la ciencia, su naturaleza interdisciplinaria permite el estudio integral del sistema mediante una descripción interna del mismo, esencialmente estructural, en términos de las variables de estado y de su interdependencia, y la descripción externa que es funcional, centrada en la conducta del sistema en términos de su interacción con el medio ambiente.

Por otra parte, la Teoría de Sistemas aporta un planteamiento de naturaleza holística e interdisciplinaria para el estudio de sistemas complejos, como lo son las organizaciones productoras de servicios sociales, cuyos problemas encierran interrelaciones de numerosos elementos. Esta teoría se sustenta en una visión orgánica del mundo como una gran organización y se opone al concepto mecanicista del mismo. Como plantea Morín (1995), hay complejidad dondequiera que se produzca un enmarañamiento de acciones y de retroacciones. Destaca que la primera complejidad es el que "nada está realmente aislado en el universo y todo está en relación". En el universo todo está en relación, es imposible conocer el todo si no se conocen las partes y viceversa. No sólo la parte está en el todo, sino el todo en las partes. Cada parte conserva su singularidad pero también contiene el todo. Cuando el pensamiento aparta el objeto de su entorno, entonces es disyuntivo y reductor porque se elimina el problema de la complejidad. La ciencia clásica propicia los estudios parcelarios que desintegran el problema, incluso al ser humano. Este pensamiento que reduce, separa y simplifica, refleja el predominio de un paradigma que nos indica qué ver y ocultamos lo que nos impone no ver. En la ciencia clásica no existe el desorden, sin embargo, el universo es fruto del diálogo entre el orden y el desorden.

El sistema -según Morín- es parte de un polisistema rodeado por un ecosistema, por ello es imperativo el desarrollo del pensamiento complejo porque permite comprender la multidimensionalidad. La complejidad proviene de la existencia de fenómenos aleatorios que no se pueden determinar y que agregan incertidumbre al pensamiento. No obstante se debe trabajar con ella para enfrentarla y manejarla. De aquí la utilidad de reemplazar la idea de objeto que es cerrada, monótona, uniforme, por la idea de sistema dotado de algún tipo de organización. 
La organización para Morín (1995) es lo que liga a un sistema. El todo tiene una cantidad de propiedades y cualidades que no tienen las partes separadas; tales cualidades nacen a nivel del todo, emergen por lo que pueden llamarse emergencias. En este sentido y de acuerdo con Morín, el todo, que en este caso sería la organización que produce servicios sociales, es más que la suma de sus elementos constitutivos y, al mismo tiempo, es menos que la suma porque la organización de un todo impone constricciones e inhibiciones a las partes que lo forman, que ya no tienen libertad. De aquí la utilidad de entender que la organización de cualquier todo o sistema, enfrenta a una complejidad conceptual y se deben observar cuáles son las ventajas y las contricciones que impone. Lo expuesto significa que toda organización que produce servicios sociales es viva, puede desarrollarse y también se degrada, tolera el desorden como elemento necesario en los procesos de creación e invención.

En relación con la naturaleza del sistema de organización de los servicios sociales, los aportes de la Teoría general de Sistemas ayudan a distinguir que ésta es definible por su cohesión, es decir, por las interrelaciones entre sus elementos componentes. Tales interacciones no se ven o perciben nunca directamente, sino que son construcciones conceptuales. Por tanto, se puede afirmar que este tipo de organización es un sistema conceptual que corresponde a una realidad y de cuyas interacciones se configuran diversos niveles de complejidad que Edgar Morín (1995) identifica con los principios de complementariedad; antagonismo, autoorganizador y autoecoorganizador.

\section{La complementariedad y el antagonismo en las interacciones del sistema}

Las cualidades emergentes en el sistema como un todo se reproducen en las partes. Dichas cualidades son: unidad global, organización, emergencia de distintos niveles, coacción e inhibición, complementariedad, antagonismo y complejidad. Lupasco (citado en Morín:1995) señala que, con el fin de que un sistema pueda formarse y existir, es necesario que todo conjunto por su propia naturaleza y por las leyes que lo rigen, sean susceptibles de acercarse, a la vez que se excluyen, o sea, de atraerse y repelerse, de asociarse y disociarse, de integrarse y desintegrarse. 
Entonces, toda interrelación del sistema se rige por el principio de la complementariedad y el principio de antagonismo. Los equilibrios organizativos son equilibrios de fuerzas antagónicas. La complejidad del sistema crea y a la vez rechaza el antagonismo. Las complementariedades sistémicas son indisociables de los antagonismos.

\section{La antiorganización o desintegración}

La antiorganización es a la vez necesaria y antagónica a la organización. Todo sistema lleva el antagonismo en su interior, lleva por tanto su propia desintegración potencial y le condena a desaparecer.

La única posibilidad de luchar contra la desintegración consiste en:

a) Integrar y utilizar al máximo los antagonismos de forma organizativa.

b) Renovar la energía y la organización potenciándola en su entorno.

c) Ser capaz de autorreorganizarse y autodefenderse.

Estas estrategias dan origen al principio autoorganizador.

\section{La autoorganización}

Se basa en el acoplamiento de un dispositivo generativo (el control de la información) y un dispositivo fenoménico que reacciona directamente al ecosistema y realiza los intercambios.

La autoorganización significa:

a) Organización de la variedad (jerarquía, diferenciación, especialización, comunicación).

b) Autorregulación (realimentación, negantropía, homeostasis).

c) Multistasia (aptitud para aceptar como satisfactorio un gran número de estados diversos).

d) Equifinalidad (alcanzar un fin por medios distintos).

e) Multifinalidad (actividades dedicadas a numerosas finalidades).

f) Aptitud para el desarrollo y la evolución. 


\section{La autoecoorganización}

El sistema autoorganizador construye su autonomía mediante la alianza del determinismo interno y de la libertad propia. La relación ecológica es organizadora. El sistema cuanto más complejo, más absorbe de su entorno, y cuanto más autónomo, es más dependiente del ecosistema, dada la multiplicidad de relaciones vitales. Las organizaciones productoras de servicios sociales son sistemas autoecoorganizativos, o sea, manejan la multiplicidad de relaciones en su dimensión microorganizacional y contextual, relaciones que se caracterizan por el antagonismo y la complementariedad y por la generación de fuerzas para la renovación de energías y potencialización de las oportunidades del entorno para autoorganizarse y "encajar" en el ecosistema.

Entonces, podemos comprender que la capacidad de la organización en autorreconstruirse está altamente vinculada al potencial humano que posee. De allí nos parece pertinente señalar que el constructivismo ${ }^{2}$ se constituye en un pilar epistemológico -teórico significativo para hacer gerencia de organizaciones productoras de servicios sociales, en tanto que el modo de pensar constructivista parte de que (Glasersfeld, 1995).

a) La viabilidad del conocimiento supone el criterio de los otros y por lo tanto los otros son importantes.

b) El conocimiento se logra en una dirección interactiva y participativa.

c) El conocimiento viable es el que encaja a los propósitos de la intervención.

d) Se conoce si se está involucrado en la situación.

Este parte de que la realidad es una construcción del mundo de la vida cotidiana, que surge de las capacidades interpretativas de los directamente implicados en ella. De aquí lo que se conoce no es el mundo en sí, sino el mundo con el conocimiento de quien observa. Lo expuesto significa que una organización que produce servicios sociales (en cuanto a su misión, objetivos, estructura organizativa,

2 Para ampliar sobre el constructivismo, consúltese Piaget, Bateson, Von Foerster, Von Glasersfeld, Watzlawick, Maturana, Varela y Flores. 
tecnologías de gestión e intervención, procesos organizativos, entre otros), es el resultado de las interpretaciones que cotidianamente realizan las personas miembras responsables de su conducción y operacionalización, de la misión y de cómo poner en práctica procesos de trabajo que permitan su alcance. Es el resultado de las interpretaciones hechas en determinadas condiciones de tiempo y de lugar, por tanto su estudio y comprensión también es el resultado de sus estructuras mentales y de sus conocimientos.

En este sentido, conocer una OPSS es una traducción y una reconstrucción, es una percepción de la realidad organizativa en un momento histórico determinado, es una interpretación entre muchas posibles. Este proceso de interpretación, para que sea útil requiere de un pensamiento complejo que permita comprender la multidimensionalidad de los factores que condicionan a un sistema.

Tal y como plantea Morín (1995), pensamiento complejo es aquel que se sabe siempre local, ubicado en un tiempo y en un momento. No es completo, sabe que siempre hay incertidumbre, escapa al dogmatismo arrogante, no cae en el escepticismo resignado porque operando una ruptura total con el dogmatismo de la certeza, se lanza valerosamente a la aventura incierta del pensamiento. Abordar el estudio de OPSS como sistemas complejos, obliga a clarificar la epistemología en que se sustenta.

Bertalanffy plantea que la teoría de sistemas difiere profundamente de las epistemologías del positivismo lógico o del empirismo, aunque comparte con ellas la actitud científica. Se caracteriza esta epistemología por partir de que la investigación de todos organizados de muchas variables requiere nuevas categorías de interacción, operación, organización, entre otros, con qué enfrentarse a nuevos problemas. Parte de que la percepción no es un reflejo de las cosas reales y el conocimiento es una aproximación a la realidad o la verdad. Es una interacción entre lo conocido y el que conoce y por tanto dependiente de una multiplicidad de factores de orden biológico, psicológico, cultural y lingüístico (Morín, 1995).

En síntesis, la Teoría General de Sistemas es tanto un modelo de ciertos aspectos generales de la realidad, como una manera de ver cosas que habían pasado desapercibidas, es una máxima metodológica.

Paul Watzlawick según la entrevista hecha por Vargas (1996) plantea la existencia de realidades de primer y segundo orden; por un 
lado la realidad que nos da nuestro sistema nervioso central y la realidad de segundo orden da un sentido, un valor, una significación, esto es arbitrario, personal, no es una cosa definitiva, es una realidad construida. Así en las organizaciones productoras de servicios sociales encontramos diversas interpretaciones de lo que son, de lo que hacen y de los desafíos organizacionales. El gerente debe aprender a observarse para comprender cómo llega a determinadas decisiones y para comprender las diversas interpretaciones que coexisten con antagonismos y complementariedades entre los actores del sistema organizacional y cómo esas interpretaciones construyen "quiebres organizacionales", "estados de ánimo", "aperturas y cierres de posibilidades" en los "espacios conversacionales" (Flores, 1995).

Podemos afirmar que un gerente social que sabe leer y conducir la organización como un sistema autoecoorganizativo, debe propugnar por una comprensión de la Organización Productora de Servicios Sociales, como una construcción que surge como consecuencia de las capacidades interpretativas de quienes la crearon, los cuales conjugan diversidad de intereses, los directamente implicados en ella; y mira a los otros como seres cognoscentes, creadores de significados y acciones con sentido. Entonces, un gerente social con perspectiva constructivista constituye su interpretación de la organización, la cual también es sólo una de las múltiples interpretaciones posibles.

Las organizaciones son parte del mundo que vivimos y que configuramos con otros, somos generadores del mundo, entonces las organizaciones son un estar siendo que se manifiestan y construyen en el "lenguajear" y emocionar o sea en la palabra, la emoción y la acción (Maturana, 1997). En el conversar construimos realidad con el otro. En el conversar es constructor de realidades y configura una red de compromisos recurrentes entre las personas miembros de las organizaciones. 


\section{Bibliografía}

Bertalanffy, L. (1987). Historia y situación de la Teoría General de Sistemas. En: L. Bertalanffy y otros, Tendencias en la Teoria General de Sistemas (29-53). Madrid: Alianza Editorial.

Buckley, Walter (1987). La epistemología vista a través de la Teoría de Sistemas. En: L. Bertalanffy, Tendencias de la Teoria General de Sistemas (219-237). Madrid: Alianza Editorial.

Díaz, Polanco (1979). Contribución a la crítica del funcionalismo. En: Víctor Bravo y otros, Teoría y realidad en Marx Durkkein y Weber, 109-153. México: Juan Pablo Editor.

Flores, Fernando (1995). Creando organizaciones para el futuro. Chile: Editorial Dolmen.

Glaserfeld, Ernst (1995). La construcción del conocimiento. En: Dora Schnitman (comp.) Nuevos paradigmas, cultura y subjetividad (115142). Buenos Aires: Paidós.

Gutiérrez P., Gabriel (1984). Metodología de las ciencias sociales, (219-232). México: Editorial Harla.

Klir, George (1987). Teoría polifónica general de sistemas. En: Bertalanffy y otros. Tendencias en la Teoría General de Sistemas, (9-29). Madrid: Alianza Editorial.

Leñero, José (1975). Introducción a la Teoría General de Sistemas. En: Modelos y técnicas de sistemas aplicados a la administración de proyectos. San José: ICAP.

Maturana, Humberto (1997). El sentido de lo humano. Chile: Editorial Dolmen.

Morín, Edgar (1995). Sociología. Capítulo II - De la naturaleza de la sociedad. Madrid: Tecnos.

Morín, Edgar (1995). Epistemología de la complejidad. En: Dora Schnitman (comp.) Nuevos paradigmas, cultura y subjetividad, 421442. Buenos Aires: Paidós.

Orchard, Robert (1987). Sobre un enfoque de la Teoría General de Sistemas. En: L. Bertalanffy y otros, Tendencias en la Teoria General de Sistemas, 237-287. Madrid: Alianza Editorial.

Rey, Pedro (1975). Conceptos generales de informática. En: Modelos y técnicas de sistemas aplicados a la administración de proyectos, 65-70. San José: ICAP.

Vargas, Pedro (1996). Entrevista con Paul Watzlawick. En: Boletín de la Academia de Terapia Sistémica, 1 (2). 